\title{
An algorithm to generate high dense packing of particles with various shapes
}

\author{
Konrad Miśkiewicz ${ }^{1,}$, Robert Banasiak $^{1}$, Maciej Niedostatkiewicz ${ }^{2}$, Krzysztof Grudzień ${ }^{1}$, \\ and Laurent Babout ${ }^{1}$ \\ ${ }^{1}$ Institute of Applied Computer Science, Lodz University of Technology, ul. Stefanowskiego 18/22, \\ 90-924 Łódź, Poland \\ ${ }^{2}$ Faculty of Civil and Environmental Engineering, Gdansk University of Technology, \\ ul. Narutowicza 11/12, 80-233 Gdańsk, Poland
}

\begin{abstract}
Discrete Element Method (DEM) is one of available numerical methods to compute movement of particles in large scale simulations. The method has been frequently applied to simulate the cases of grain or bulk material as the major research issue. The paper describes a new method of generating high dense packing with mixed material of two different shape used in DEM simulation. The initial packing is an important parameter to control, because have influence on the first few seconds after start the simulation. Some-times when the material in silo is arranged with loose packing before the start, the particles move downward gravity. These changes between the start and the first few seconds in simulations act strongly on the results at the end of a discharging process in silo. At the initial simulation time it is important to prepare proper packing with mixed material, in order to make sure that the particles will not move due to gravity action. This solution is a necessary step to integrate in the simulation procedure in order to compare later the computer simulation with experimental measurements of material discharge in a silo.
\end{abstract}

\section{Introduction}

In many cases in order to validate a number of constructions and check a proper technology or construction simulations are prepared. This work deals with software which used many kind of algorithms, with mathematical equations. The major numerical methods are Finite Element Method (FEM)[1], and Discrete Element Method (DEM)[2, 3]. The first numeric method helps to prepare structural analysis, electromagnetic potential or heat transfer. The second one is used to deal with motion, dynamics of small particles in large scale simulations. This method is addressed to prepare flow granular material or particle mechanics for many of problems especially for discharging process in silos.

There is a large number of software to use numerical methods mentioned above. Both commercial and free software is at hand. The free software such like YADE [4] or WooDEM, was created by scientists who work on granular flow. This frameworks explore methods to generate spherical or ellipsoid packing[5,6]. It is important to

*E-mail: konrad.miskiewicz@p.lodz.pl 
emphasize that this method is not intended generate packing with mixed material. One of the methods was implemented, but the result was a small dense packing[7]. The algorithm was expanded on new methods especially in order to generate packing with high density mixed material. A number of algorithms to generate spherical packing, was developed[ 8,9 , 10]. One algorithm helped in large scale simulations, applying tetrahedral mesh which can be generated by other software: Ghs3D, Gmsh or Netgen[11, 12]. Another algorithm firstly creates particles at random, next starts the compression[13]. The basic method of sphere packing was called Face Centered Cubic(FCC) and Hexagonal Close Packing (HCP)[14].

\section{Mathematical formulations}

Generation of a mixed material in one simulation with high dense packing is impossible with methods described earlier. In this case the particles showed two different shapes of grains. One of them was a sphere, not difficult to be describe in simulation. This object detects only two parameters to describe its properties, the radius of sphere and sphere positioning in $3 \mathrm{D}$ space. However the spheres are packed together with tracking particles the ellipsoids. Filing with the use of the different material results in a few percentage of the whole. Description of this type of material is more difficult. As in the case of spheres, ellipsoid also shows a center saved in the same 3D space. It is important to emphasize that this shape needs more parameters to describe the object. In order to characterize the object magnitude three dimensions are required in the axes of symmetry. This is the main difference between the sphere and the ellipsoid. It is worth noticing that three axial dimensions coinciding, then the turn the ellipsoid into to sphere. Rotation acts strongly on positioning the particles in simulation. This issue is linked with the ellipsoid case, not active while spheres are taken. It is important to emphasize that the ellipsoid rotation is a new issue in high dense packing, acting on free space around this kind of particles. Rotation maybe considered in three ways: using Euler equation, rotation around axis and quaternion. In many cases the last method is a simple and straightforward way to deal with rotations, and it is more realistic than others.

In the previous proposed algorithm the dense packing is not implemented[6]. A solution was developed to assure a high level of the mixed packing. Following the methods, to generate mixed material packing, the algorithm must work in two and three dimensions. The main shape of silo prepared in simulation to packing, is divided to conduct smaller subproblems. As a result of this solution the boundaries between sub-problems were created. This method is aimed at faster computing and to improve the process of preparing simulations. On the sub-problem boundaries the method to generate high dense packing must work in two dimensions. The particles are generated in $\mathrm{x}$ and $\mathrm{y}$ axis the $\mathrm{z}$ axis is fixed. Particles are moved from free space to a position calculated by an algorithm.

Equation of an ellipsoid is given (1):

$$
\frac{x^{2}}{a^{2}}+\frac{y^{2}}{b^{2}}+\frac{z^{2}}{c^{2}}=1
$$

where:

a, b, c principal semi-axes, and $\mathrm{a}>\mathrm{b}>\mathrm{c}$.

When the $a ; b$; c dimensions are the same the ellipsoid are turns into a sphere. With the aim of ordering particles in right place, the algorithm must calculate the vector of translation. However, the points on the surface are investigated first to detect the shortest distance to point the direction of movement (Fig. 1). 


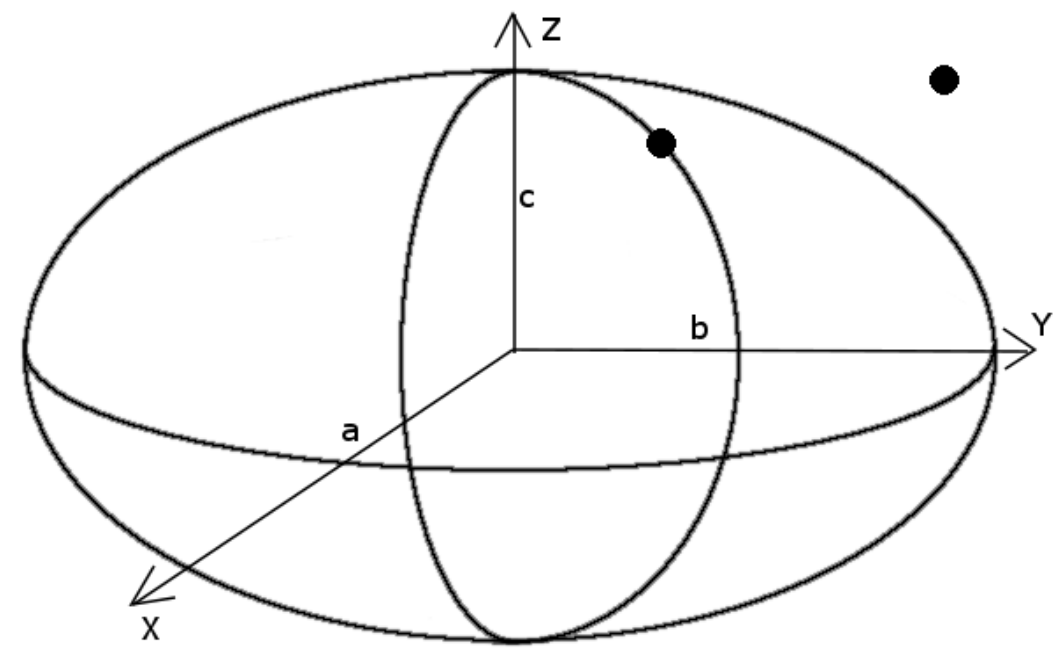

Fig. 1. Schematic picture of ellipsoid and the point on surface.

$$
\begin{aligned}
& x_{p}=\frac{a \cdot x_{L}}{\sqrt{x_{L}^{2}+y_{L}^{2}+z_{G}^{2}}} \\
& y_{p}=\frac{b \cdot y_{G}}{\sqrt{x_{L}^{2}+y_{L}^{2}+z_{L}^{2}}} \\
& z_{p}=\frac{c \cdot z_{G}}{\sqrt{x_{L}^{2}+y_{L}^{2}+z_{L}^{2}}}
\end{aligned}
$$

where:

$$
\begin{aligned}
& x_{p v y_{p}, Z_{p}} \quad \text { the coordinate of point on the surface on ellipsoid, } \\
& x_{G}, y_{G}, \mathbb{Z}_{G} \quad \text { the point of the particle moved (translated). }
\end{aligned}
$$

The vector of translation was calculated by simple equations:

$$
\overrightarrow{P G}=\left(x_{G}-x_{p v} y_{G}-y_{p}, z_{G}-z_{p}\right)
$$

The equations above are used to change the position of a particle to the nearest boundary of shape, identified by a silo construction. According that ellipsoid could rotation matrix is introduced in the equation. After that the view of equation is presented below (6):

$$
\left[\begin{array}{l}
x_{p} \\
y_{p} \\
z_{p}
\end{array}\right]_{\text {True }}=\left[\begin{array}{l}
x_{0} \\
y_{0} \\
z_{0}
\end{array}\right]+R\left[\begin{array}{l}
x_{p} \\
y_{p} \\
y_{p}
\end{array}\right]_{\text {Standartd }}
$$

While $x_{0}, y_{0}, z_{0}$ marks the centre of an ellipsoid, $R$ is the rotation matrix, and standard matrix means that the point is not rotated. 
In addition to positioning particles near the boundary of sub-problems, remember that in the middle of silo or sub-problems the material is intended to detect proper ordering. Each particle must show at least two contact points. In order to calculate the vector of particle translation different equations are applied. The change related to the point is not situated on construction surface of silo, but on the centre of the second particles (Fig.2).

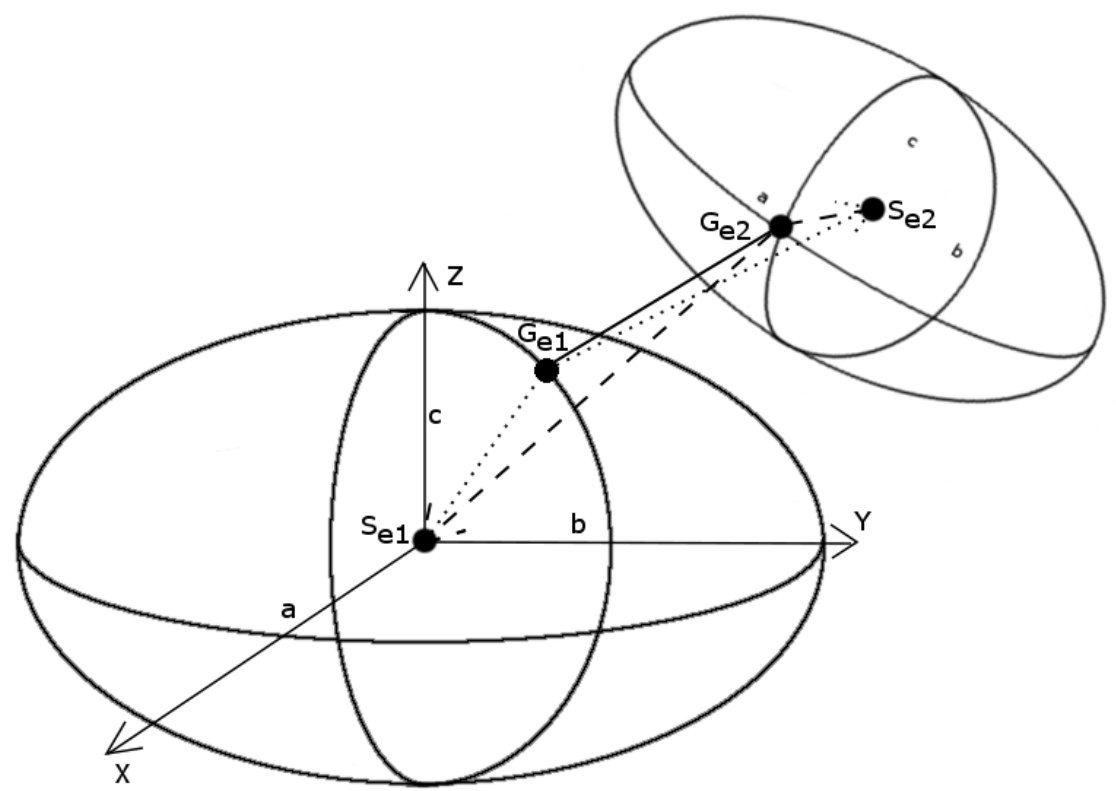

Fig. 2. Schematic picture of two ellipsoid.

\section{Algorithm}

Some of algorithms generate only one type of material, sometimes the process of filling the model in simulations relies on the positioning shapes in right place. This algorithm is not finally efficient, because while the solution is prepared it is not matched to real result shown a high difference. The work is focused on implementing some features to generating mixed material with high dense packing.

In the first step trust the users describe the layout of sphere or ellipsoid and set the ratio parameter, to describe the proportion between this two material types. Of course add information is required about the object to be filled, for example silo. Next the algorithm calculates the boundaries and execute, the procedure to partition the problem to smaller ones. After that the first particle is generated, and checked that the object is in the silo or shape to be filled with material. If the shape lies in the boundaries, the vector translation is computed to move particle to the boundaries. Before the position changes, an algorithm computes and checks the way to all of boundaries in sub-problems. The nearest way is chosen, so the centre of sphere or ellipsoid is changed.

The next step is to generate random particles whose position is located in the silo. When on entire was entered in the simulation, it started the proper process of ordering particles. The first object that moved to boundaries at the beginning of the process was marked the major one main in this sub-problem or on the boundaries. While changing the position of particles to right places, the method must calculate the vector of translation. It is emphasized that each material shows different directions of movement and different distance to change. In 
the first step of this complex process is to search nearest particles and then calculate the vector of translation. Of course during this stage, it is checked that the particle does not crossed the boundaries of the silo. When the position of two shapes was changed, algorithm calculate the distance between them to verify the collision. To protect count of spheres or ellipsoids around actually working particle, method calculate the angle ensuing on the main particle in sub-problems or in boundaries. During this process and if all of condition was pass, are saved with map statement, where inform that main particle have this neighbours, and vice versa. Of course the shapes which was moved to the main particle have only one or two neighbours. After this the algorithm change object of working for whom checked next random particles and calculated new vectors of move. The process of computing moves and conditions are repeated for all of material which are in filled object in simulation, to moment when the packing are high dense. At the end if have more space algorithm generate additional particles which was moved immediately to right place.

Above description the method that work on boundary, and on two dimensional area. In three dimension view this process is the same, but all of this particle have big priority to move not in $\mathrm{x}$ and $\mathrm{y}$ axis but in $\mathrm{z}$ axis. It is important because after start the simulation and add gravity, the particles do not moved to bottom in silo. Of course the boundary which generated earlier in this process was mixed and also moved to down, and changed to irregular surface, but this change is very low. 


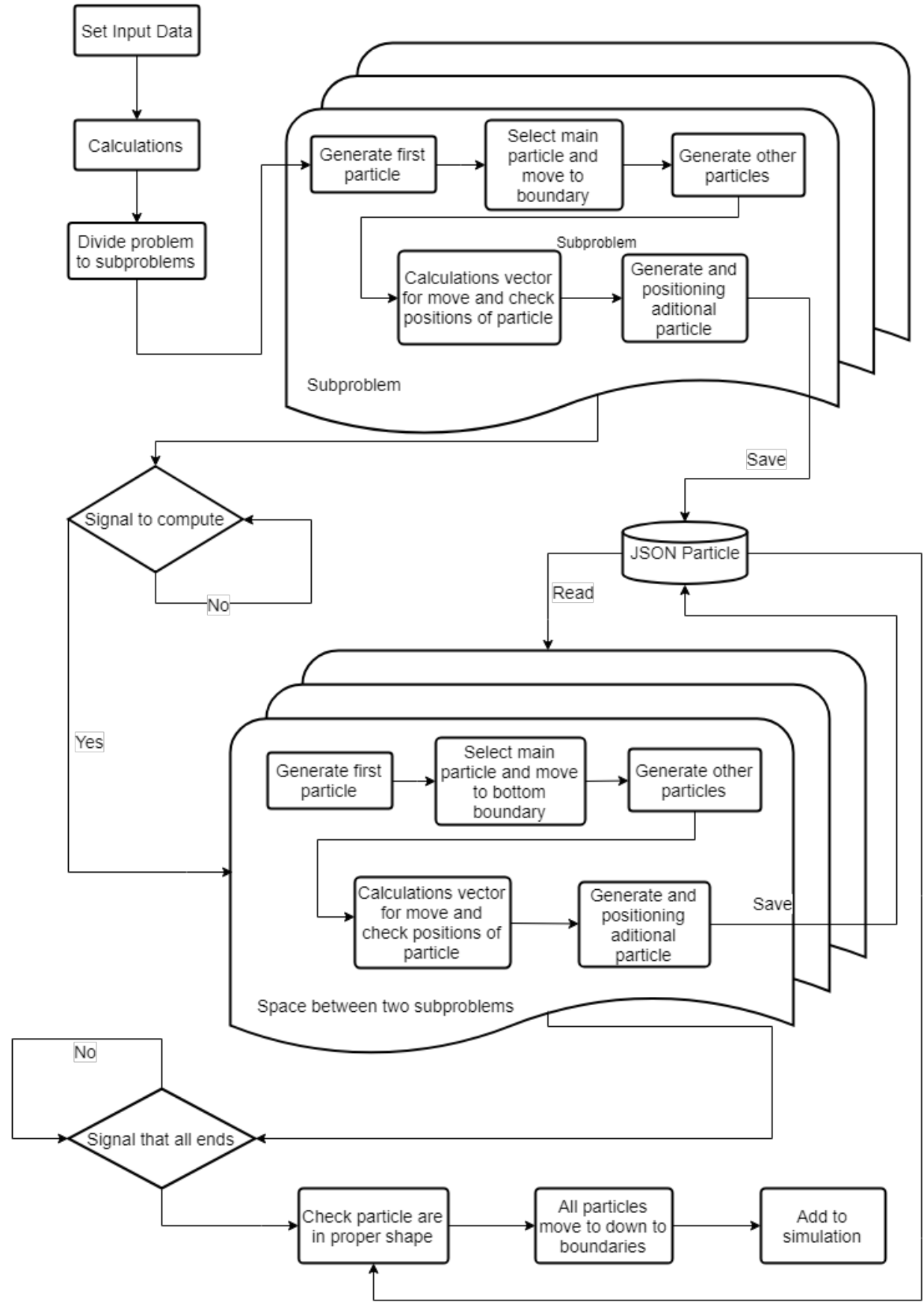

Fig. 3. Schematic picture with algorithm. 


\section{Results Analysis}

Most of algorithm for generate packing with sphere the dense packing is on level average $0: 74$. It is interesting that some of methods are low level, because one of them generate random packing and other calculates the possible max arrangement of sphere in filled object. It is important to emphasize that generating packing with ellipsoid give different dense, which average is on 0:75. This results is not different as much but the shape of particle are very different, and have influence of dense packing.

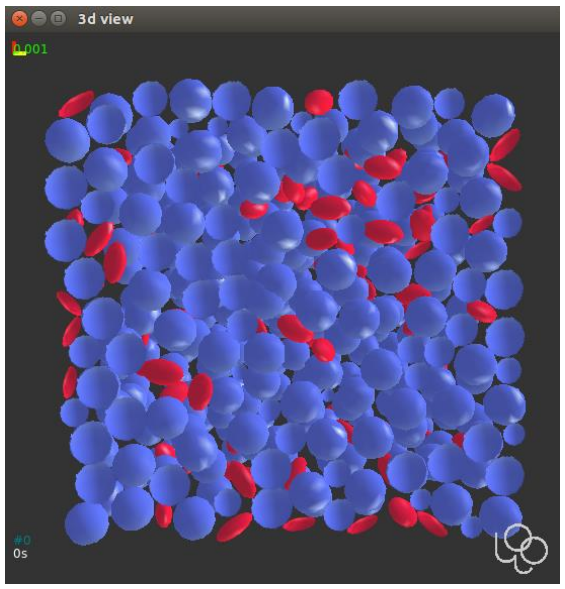

Fig. 4. Schematic picture of packing mixed material of two different shapes.

The proposal algorithm generate dense packing with mixed material of two different shapes, especially with sphere and ellipsoid in one object. It is important to set the ratio between particles that generated in schematic chart about dense packings that the mixed material with two different shapes have influence to the dense packing (Fig. 5). When in the object which filled have only sphere, the ratio is average $0: 70$. This result is very good for random packing to compare with other algorithms. In case with mixed material this results reaches average about 0:64 for the coefficient of ratio in $25 \%$. It is important to emphasize that this result is for mixed material with two different shape, what means that is very good for the above case.

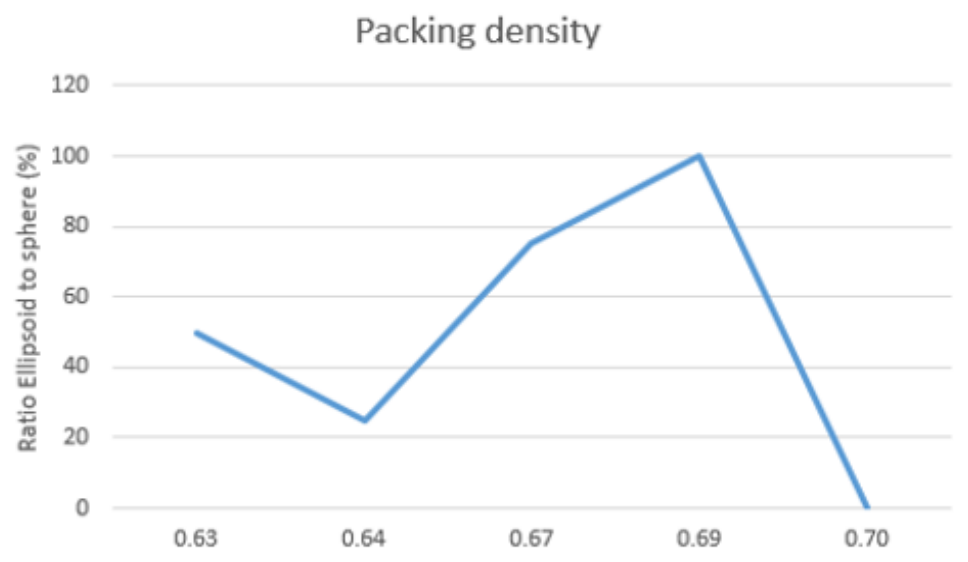

Fig. 5. Schematic chart about dense packing. 


\section{Conclusion}

The algorithms and methods that was proposed for generating particles in high dense packing is not effective with mixed material. Proposal algorithm allow to compute mathematical equations which are helped to positioning mixed material. It is emphasize that this method are used not only in generating particles, but also when the material was saved in database to checking the collision and property of dense packing. Division this problem to smaller one was good efficient, because most of calculation are starting parallel. This solution allow to save time for computing and preparing simulations and are used all power that was in computer. Finally this method helped to compare the real results, which was conducted in X-ray laboratory, with computer simulation.

\section{References}

1. P. Wriggers, Nonlinear Finite Element Methods (Springer, Berlin Heidelberg, 2008)

2. H. K. Dang, M. Meguid, International Journal of Geomechanics, 10, 85-91 (2010)

3. M. Jiang, H. Yu, Modern Trends in Geomechanics, 241-269 (2006)

4. J. Kozicki, F. V. Donzé, Computer Methods in Applied Mechanics and Engineering, 197, 4429-4443 (2008)

5. A. Doney, F. H. Stillinger, P. M. Chaikin, S. Torquato, Physical Review Letters, 251, 255506-1 (2004)

6. R. K. McGEARY, Journal of the American Ceramic Society, 44 (10), 513-522 (1961)

7. K. Miśkiewicz, R. Banasiak, M. Niedostatkiewicz, K. Grudzień, L. Babout, Proceedings of International Interdisciplinary PHD Workshop, 366-371 (2017)

8. K. Bagi, Granular Matter, 7 (1), 31-43 (2005)

9. B. Harthong, J. F. Jérier, P. Dorémus, D. Imbault, F. V. Donzé, International Journal of Solids and Structures, 46, 3357-3364 (2009)

10. R. R. Valera, I. P. Morales, S. Vanmaercke, C. R Morfa, L. A. Cortés, H. D. G. Casañas, Computational Particle Mechanics, 2 (2), 161-172 (2015)

11. J. F. Jerier, V. Richefeu, D. Imbault, F. V. Donzé, Computer Methods in Applied Mechanics and Engineering, 199, 1668-1676 (2010)

12. J. F. Jerier, D. Imbault, F. V. Donze, P. Doremus, Granular Matter, 11 (1), 43-52 (2009)

13. B. D. Lubachevsky, F. H. Stillinger, Journal of Statistical Physics, 60, 561-583 (1990)

14. J. Conway, J. A. Sloane Neil, Sphere packings, lattices and groups (Springer Science+Buisnes Media LLC, New York, 1991) 